\section{Has COVID-19 Delayed the Diagnosis and Worsened the Presentation of Type 1 Diabetes in Children?}

https://doi.org/10.2337/dc20-1321
Ivana Rabbone, ${ }^{1}$ Riccardo Schiaffini, ${ }^{2}$ Valentino Cherubini, ${ }^{3}$ Claudio Maffeis, ${ }^{4}$ Andrea Scaramuzza, ${ }^{5}$ and the Diabetes Study Group of the Italian Society for Pediatric Endocrinology and Diabetes*

\section{OBJECTIVE}

To evaluate whether the diagnosis of pediatric type 1 diabetes or its acute complications changed during the early phase of the coronavirus disease 2019 (COVID-19) pandemic in Italy.

\section{RESEARCH DESIGN AND METHODS}

This was a cross-sectional, Web-based survey of all Italian pediatric diabetes centers to collect diabetes, diabetic ketoacidosis (DKA), and COVID-19 data in patients presenting with new-onset or established type 1 diabetes between 20 February and 14 April in 2019 and 2020.

\section{RESULTS}

Fifty-three of 68 centers $(77.9 \%)$ responded. There was a $23 \%$ reduction in new diabetes cases in 2020 compared with 2019. Among those newly diagnosed patient who presented in a state of DKA, the proportion with severe DKA was $44.3 \%$ in 2020 vs. $36.1 \%$ in $2019(P=0.03)$. There were no differences in acute complications. Eight patients with asymptomatic or mild COVID-19 had laboratory-confirmed severe acute respiratory syndrome coronavirus 2 .

\section{CONCLUSIONS}

The COVID-19 pandemic might have altered diabetes presentation and DKA severity. Preparing for any "second wave" requires strategies to educate and reassure parents about timely emergency department attendance for non-COVID-19 symptoms.

At its peak, coronavirus disease 2019 (COVID-19) significantly reduced pediatric emergency department (ED) access, most likely due to the fear of infection (1). During the pandemic, the International Society for Pediatric and Adolescent Diabetes received information from its members about several new type 1 diabetes cases or diabetic ketoacidosis (DKA) episodes where hospitalization was delayed due to the closure of non-COVID-19 services (2). There were also concerns that parents had delayed seeking timely medical advice for children with symptoms of diabetes or DKA, resulting in increased numbers of presentations of severe DKA. Based on these anecdotal cases in Italy and other countries (2), we hypothesized that the COVID-19 pandemic might have affected the number of new diabetes diagnoses and the severity of DKA. We therefore conducted a survey to evaluate whether the diagnosis of pediatric type 1 diabetes or its acute complications changed during the early phase of the COVID-19 pandemic in Italy compared with the same period in 2019.
${ }^{1}$ Division of Pediatrics, Department of Health Sciences, University of Piemonte Orientale, Novara, Italy

${ }^{2}$ Diabetes Unit, Bambino Gesù Children's Hospital, Rome, Italy

${ }^{3}$ Department of Women's and Children's Health, G. Salesi Hospital, Azienda Ospedaliero-Universitaria Ospedali Riuniti, Ancona, Italy

${ }^{4}$ Pediatric Diabetes and Metabolic Disorders Unit, Regional Center for Pediatric Diabetes, University City Hospital of Verona, Verona, Italy

${ }^{5}$ Division of Pediatrics, Azienda Socio Sanitaria Territoriale Cremona, Ospedale Maggiore di Cremona, Cremona, Italy

Corresponding author: Andrea Scaramuzza, andrea .scaramuzza@asst-cremona.it

Received 1 June 2020 and accepted 10 July 2020

This article contains supplementary material online at $h$ ttps://doi.org/10.2337/figshare.12675089.

A complete list of the members of the Diabetes Study Group of the Italian Society for Pediatric Endocrinology and Diabetes can be found in the supplementary material online.

This article is part of a special article collection available at https://care.diabetesjournals.org/ collection/diabetes-and-COVID19.

(C) 2020 by the American Diabetes Association. Readers may use this article as long as the work is properly cited, the use is educational and not for profit, and the work is not altered. More infor-

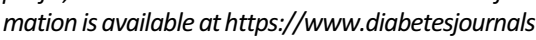
.org/content/license.

See accompanying article, p. XXX. 


\section{RESEARCH DESIGN AND METHODS}

This was a cross-sectional electronic survey of all 68 Italian pediatric diabetes centers (3), including all tertiary referral centers in Italy. Each center was asked to review all records of patients $<15$ years of age with recorded onset of diabetes between 20 February 2020 and 14 April 2020 and during the same period in 2019. The survey was Web based with data collection centralized at the Department of Health Sciences, University of Piemonte Orientale.

Each center provided the number of patients diagnosed with type 1 diabetes, how many had DKA and its severity, how many were diagnosed with COVID-19, and how many patients with established diabetes had DKA or severe hypoglycemia.

Type 1 diabetes was defined according to autoantibody positivity. DKA was defined according to International Society for Pediatric and Adolescent Diabetes criteria (blood glucose $>11 \mathrm{mmol} / \mathrm{L}$ [ $\sim 200 \mathrm{mg} / \mathrm{dL}$, venous $\mathrm{pH}<7.3$ or bicarbonate $<15 \mathrm{mmol} / \mathrm{L}$, ketonemia and ketonuria), with DKA severity categorized as follows: mild, venous $\mathrm{pH}<7.3$ or bicarbonate $<15 \mathrm{mmol} / \mathrm{L}$; moderate, $\mathrm{pH}$ $<7.2$, bicarbonate $<10 \mathrm{mmol} / \mathrm{L}$; and severe, $\mathrm{pH}<7.1$, bicarbonate $<5 \mathrm{mmol} / \mathrm{L}$ (4). Severe hypoglycemia was defined as an episode with loss of consciousness or requiring hospitalization.

The $\chi^{2}$ test was used to evaluate differences between 2020 and 2019 data. $P<0.05$ was deemed statistically significant. Statistical analyses were performed using SPSS Statistics, version 23 (IBM Statistics, Chicago, IL).

\section{RESULTS}

Of the 68 centers belonging to the Italian Society for Pediatric Endocrinology and Diabetes, 53 (77.9\%) completed the Web-based survey and returned complete data, which included all diabetes tertiary referral centers $(n=39)$ in Italy. The results of the survey are shown in Table 1. Between 20 February 2020 and 14 April 2020, 160 children aged 014 years were diagnosed with type 1 diabetes-23\% lower than in 2019. DKA of any severity was observed in 61 of 160 patients (38.1\%) in 2020 and in 86 of 208 patients in 2019 (41.3\% [not significantly different]). Whereas the overall proportion of patients with newly diagnosed diabetes with severe DKA was similar in 2020 and 2019 (16.9\% vs. $14.9 \%$, respectively), among all patients who presented in a state of DKA, the proportion with severe DKA was $44.3 \%$ in 2020 vs. $36.1 \%$ in $2019(P=0.03)$ (Table 1 ).

DKA episodes and severe hypoglycemia requiring hospitalization in patients with established diabetes were similar between the two periods, although there were slightly fewer cases in 2020 (13 DKA episodes in 2020 vs. 22 in 2019 and 10 severe hypoglycemia cases in 2020 vs. 13 in 2019).

During the observation period, only eight patients were diagnosed with COVID-19 (range 6-16 years of age): one at diabetes onset and seven patients with established diabetes (diabetes for 1-11 years), all of whom were asymptomatic $(n=4)$ or had only mild symptoms ( $n=$ 4). All had nasopharyngeal swabs positive for severe acute respiratory syndrome coronavirus 2 (SARS-CoV-2) by RT-PCR; one also had confirmatory serological detection of SARS-CoV-2-specific antibodies.

\section{CONCLUSIONS}

In this study, the Diabetes Study Group of the Italian Society for Pediatric Endocrinology and Diabetes investigated whether COVID-19 affected children with new-onset and existing type 1 diabetes in the first 2 months of the pandemic in Italy. During the observation period, there were $23 \%$ fewer new diabetes cases compared with the same period in 2019, and children presenting with DKA had more severe DKA $(\mathrm{pH}<7.1)$ in 2020 than in 2019 (44.3\% vs. $36 \%$, respectively; $P=0.03$ ). The lower number of new-onset diabetes cases during the observation period might be due to not only a fear of SARS-CoV-2 exposure but also lower exposure to seasonal viruses. Children stopped going to school on 20 February 2020, thus reducing interpersonal interactions and the chance of acquiring seasonal viruses, which are a known precipitating factor for type 1 diabetes. The impact of school closures, quarantine, and lack of exposure to seasonal viruses on future rates of diabetes will be important to quantify.

We did not observe a higher incidence of acute complications of diabetes during the first 40 days of the pandemic, with fewer DKA and severe hypoglycemia cases than during the same period the previous year. Perhaps contributing to this, Tornese et al. (5) reported that glycemic control of type 1 diabetes in adolescents using hybrid closed-loop systems did not worsen during the COVID19 restrictions and actually improved in those who continued physical activity during the quarantine. It is also possible that being quarantined, the fear of becoming ill, and the presence of parents/ guardians at home improved self-care and increased the supervision of diabetes control.

Although children are generally less prone to COVID-19 and have a milder disease course, children with existing comorbidities could remain at higher risk of complications (6). Adults with diabetes are at high risk of complications and death from COVID-19 $(7,8)$, but to our knowledge there are no equivalent data for children with diabetes. Furthermore, it is possible that SARS-CoV-2 might itself be diabetogenic, similar to what was observed in patients with severe acute

Table 1-Diabetes onset, DKA at onset, and acute complications in the pediatric population (0-15 years) observed during COVID-19 pandemic (20 February-14 April 2020) compared with the same period in 2019

\begin{tabular}{|c|c|c|c|c|c|c|}
\hline & \multicolumn{2}{|c|}{2020} & \multicolumn{2}{|c|}{2019} & \multirow[b]{2}{*}{$\Delta$} & \multirow[b]{2}{*}{$P$} \\
\hline & $n$ & $\%$ & $n$ & $\%$ & & \\
\hline Patients with diabetes onset & 160 & - & 208 & - & -48 & - \\
\hline Patients with DKA (pH <7.3) & 61 & 38.1 & 86 & 41.3 & -3.2 & 0.08 \\
\hline Patients with severe DKA ( $\mathrm{pH}<7.1$ ) & 27 & 16.9 & 31 & 14.9 & $2.0 \%$ & 0.09 \\
\hline Proportion of DKA patients with severe DKA & 27 & 44.3 & 31 & 36.0 & $8.3 \%$ & 0.03 \\
\hline DKA episodes in patients with established diabetes & 13 & - & 22 & - & -9 & - \\
\hline $\begin{array}{l}\text { Severe hypoglycemia episodes in patients with } \\
\text { established diabetes }\end{array}$ & 10 & - & 13 & - & -3 & - \\
\hline
\end{tabular}

Severe hypoglycemia was defined as an episode with loss of consciousness or requiring hospitalization. The value in boldface type represents significant difference between groups. 
respiratory syndrome coronavirus 1 pneumonia (9) - an association that requires further study in both adults and children.

Only eight of our patients had laboratory confirmation of SARS-CoV-2 infection. These patients were tested due to having mild COVID-19 symptoms or because they lived close to a known positive patient. These low numbers seem to support the evidence that children are generally less prone to COVID19 and have a milder disease course (6,10-12).

As the pandemic continues, further work is necessary to explore how this disease affects children to develop bestevidence guidelines and optimal care for our vulnerable patients (11). In the absence of guidelines, the main strategy for reducing the risk and severity of SARSCoV-2 infection in children with diabetes seems to be optimizing glycemic control $(5,6)$.

Judicious access to pediatric EDs at any time is needed to avoid severe consequences. A recently published survey of pediatric EDs in several European countries found variability and gaps in preparedness and responses to the COVID-19 pandemic (13). A secure non-COVID-19 path through pediatric EDs is essential to help and reassure parents bringing their children to the ED with other conditions such as diabetes. Specific awareness campaigns could be of help in these difficult times.

Our study is limited by only comparing data across two consecutive years, so we cannot rule out that the 2020 decrease might reflect random variation. Nevertheless, taken together with overall changes in pediatric ED attendance figures during the pandemic (1), these results should regardless prompt forward planning to mitigate the effect any "second wave" might have on non-COVID-related disease.

\section{Conclusion}

These data suggest that during the COVID19 pandemic a good number of children received a delayed diagnosis, altering the presentation of type 1 diabetes and severity of DKA. In preparation for any potential "second wave," specific strategies are essential to educate and reassure parents about timely attendance at the ED for children with symptoms that are not related to COVID-19.

Duality of Interest. No potential conflicts of interest relevant to this article were reported. Author Contributions. I.R. and A.S. conceptualized and designed the study, designed the data collection instrument, collected and analyzed data, wrote the manuscript, and contributed to discussions. R.S., V.C., and C.M. supervised data collection and analysis and critically revised and reviewed the manuscript. Members of the Diabetes Study Group of the Italian Society for Pediatric Endocrinology and Diabetes collected data and discussed and commented on data analyses. A.S. is the guarantor of this work and, as such, had full access to all the data in the study and takes responsibility for the integrity of the data and the accuracy of the data analysis.

\section{References}

1. Scaramuzza A, Tagliaferri F, Bonetti L, et al. Changing admission patterns in paediatric emergency departments during the COVID-19 pandemic. Arch Dis Child 2020;105:704-706

2. Coronavirus infection (COVID-19)-II ISPAD summary, 2020. Accessed 23 June 2020. Available from https://www.ispad.org/page/CoronavirusinfectionCOVID-19-IIISPADSummary.

3. Giorgetti C, Ferrito L, Zallocco F, lannilli A, Cherubini V; Study Group for Diabetes of ISPED.
Organization and regional distribution of centers for the management of children and adolescents with diabetes in Italy. Ital J Pediatr 2015;41:7477

4. Wolfsdorf JI, Allgrove J, Craig M, et al.; International Society for Pediatric and Adolescent Diabetes. ISPAD Clinical Practice Consensus Guidelines 2014. Diabetic ketoacidosis and hyperglycemic hyperosmolar state. Pediatr Diabetes 2014; 15(Suppl. 20):154-179

5. Tornese G, Ceconi V, Monasta L, Carletti C, Faleschini E, Barbi E. Glycemic control in type 1 diabetes mellitus during COVID-19 quarantine and the role of in-home physical activity. Diabetes Technol Ther 2020;22:462-467

6. Ludvigsson JF. Systematic review of COVID-19 in children shows milder cases and a better prognosis than adults. Acta Paediatr 2020;109: 1088-1095

7. Yang J, Zheng $Y$, Gou X, et al. Prevalence of comorbidities in the novel Wuhan coronavirus (COVID-19) infection: a systematic review and meta-analysis. Int J Infect Dis 2020;94:91-95

8. Grasselli G, Zangrillo A, Zanella A, et al.; COVID-19 Lombardy ICU Network. Baseline characteristics and outcomes of 1591 patients infected with SARS-CoV-2 admitted to ICUs of the Lombardy Region, Italy. JAMA 2020;323:15741581

9. Rubino $F$, Amiel SA, Zimmet $P$, et al. Newonset diabetes in Covid-19. N Engl J Med. 12 June 2020 [Epub ahead of print]. DOI: 10.1056/ NEJMc2018688

10. Ho C, Ng NBH, Lee YS. Caring for pediatric patients with diabetes amidst the Coronavirus Disease 2019 storm. J Pediatr 2020;223:186187

11. Dayal D. We urgently need guidelines for managing COVID-19 in children with comorbidities. Acta Paediatr 2020;109:1497-1498

12. Mustafa NM, A Selim L. Characterisation of COVID-19 pandemic in paediatric age group: a systematic review and meta-analysis. J Clin Virol 2020;128:104395

13. Bressan S, Buonsenso D, Farrugia R, et al. Preparedness and response to Pediatric CoVID19 in European Emergency Departments: a survey of the REPEM and PERUKI networks. Ann Emerg Med. 15 May 2020 [Epub ahead of print]. DOI: 10.1016/j.annemergmed.2020. 05.018 